\title{
Can we move photons?
}

\author{
Oleg L. Berman ${ }^{1}$, Roman Ya. Kezerashvili ${ }^{1,2}$, and Yurii E. Lozovik ${ }^{3,4}$ \\ ${ }^{1}$ Physics Department, New York City College of Technology, The City University of New York, Brooklyn, NY 11201, USA \\ ${ }^{2}$ The Graduate School and University Center, The City University of New York, New York, NY 10016, USA \\ ${ }^{3}$ Institute of Spectroscopy, Russian Academy of Sciences, 142190 Troitsk, Russia \\ ${ }^{4}$ Moscow Institute of Physics and Technology (State University), 141700, Dolgoprudny, Russia
}

(Dated: November 6, 2018)

\begin{abstract}
The drag effects in the system of spatially separated electrons and excitons in coupled quantum wells (CQWs) embedded in an optical microcavity are predicted. It is shown that at low temperature an electron current induces the polariton flow, therefore, a transport of photons along the cavity. However, the superfluid polariton component does not contribute to the electron drag. The polariton-electron at the low temperatures and exciton-electron at the high temperatures drag coefficients are presented. It is shown that the drag coefficients increase when temperature increases. We discuss possible experiments for the observation of the electron-polariton drag effect.
\end{abstract}

Key words: Drag effect; Exciton-polaritons; Superfluidity

PACS numbers: 71.36.+c, 71.35.-y, 03.75.Kk, 73.21.Fg

Recently properties of $2 \mathrm{D}$ excitonic polaritons [1] in a quantum well (QW) embedded in an optical microcavity have been the subject of theoretical and experimental studies (see Refs. 22, 3] and references therein). The system under study consists of two Bragg mirrors, forming an optical microcavity, and quantum well embedded within the cavity at the antinodes of the confined optical mode. The resonant exciton-photon interaction results in the Rabi splitting in the excitation spectrum and formation of upper and lower polaritons, which are the superpositions of the excitons and cavity photons. It was assumed that the modes of the microcavity photon and exciton in a $\mathrm{QW}$ were in resonance at $k_{\|}=0$, where $k_{\|}$is the component of the wave vector along the microcavity. The extremely small effective mass of the lower polariton results in a high critical temperature for superfluidity [4, 5]. Remarkable success was achieved in the observation of condensation, coherent effects and anomalous transport properties in the microcavity polariton system [6 9$]$. The unambiguous, direct observation of the superfluidity of the cavity polariton system is still an actual and essential task.

In the present Letter we predict the drag effects of the cavity polaritons and electrons, and analyze the manifestation of the polariton superfluidity in these phenomena. We consider two neighboring quantum wells embedded in an optical microcavity: the first QW is occupied by $2 \mathrm{D}$ electron gas (2DEG) and the second QW is occupied by the excitons created by the laser pumping.

At low temperature $k_{B} T \ll \hbar \Omega_{R}$, where $\hbar \Omega_{R}$ is Rabi splitting, and $k_{B}$ is Boltzmann constant, and at the resonance of excitons with cavity photons, the exciton polaritons are formed by the quantum superposition of the excitons with the cavity photons [10]. By focusing laser pumping in some region of the cavity the gradient of excitons and exciton polaritons can be generated. These gradients induce the polariton and exciton currents, and in a turn the normal (non-superfluid) component of mov- ing exciton liquid drags the electrons in the neighboring QW due to the electron-exciton interaction. So the electric current would be generated by the current of the normal component of exciton polariton liquid.

In another scenario by applying electric voltage in the QW with 2DEG the electronic current is induced, and this current drags the normal (non-superfluid) component of exciton liquid in the neighboring QW. There is the quantum superposition of the excitons and the cavity photons. So the cavity photons can be dragged and, thus, controlled by the electric voltage. Besides, possible applications of the control of photons or electrons by the polariton-electron drag can be used for study of the properties and phases in the polariton and exciton system, particularly, the superfluidity of the system (in some analogy with the Coulomb drag effects in the electronhole system, see Refs. [11 15] and references therein). Interestingly enough to mention that the first time drag effects were studied in the electron-phonon system in 1945 in Ref. [16].

Note that at high temperature, $k_{B} T \gtrsim \hbar \Omega_{R}$, the polariton states are occupied mainly far from the polariton resonance, and in these states the exciton-photon quantum superposition is negligible. Thus, at high temperatures only the exciton-electron drag is essential, and the exciton current can induce the electron current as well as the electron current can produce the exciton current.

We introduce the drag coefficients $\lambda_{p}$ and $\lambda_{e x}$ for electrons in the 2DEG dragged by the moving polaritons and exitons, respectively. For the case when the electric field is applied to the system of electrons in the QW we introduce the drag coefficients $\gamma_{p}$ and $\gamma_{e x}$, respectively, for polaritons and excitons dragged by the electron current. In the two-layer system there are a current of electrons and current of polaritons or excitons. The current of polaritons or excitons is $\mathbf{i}_{i}=n_{i} \mathbf{v}_{i}$, where $n_{i}$ and $v_{i}$ are density and average velocity, and the index $i$ is defined as $i=e x$ for excitons and $i=p$ for polaritons. The electron 
current $\mathbf{j}=-e n_{e l} \mathbf{v}_{e l}$, where $n_{e l}$ is the density and $\mathbf{v}_{e l}$ is the average velocity of electrons in the electron layer. These currents can be expressed in terms of the density gradient $\nabla n_{i}$ in the polariton or exciton subsystem, drag coefficients $\lambda_{i}$ and $\gamma_{i}$, and external electric field $\mathbf{E}$ applied to the 2DEG by the following matrix expression:

$$
\left(\begin{array}{c}
\mathbf{i}_{i} \\
\mathbf{j}
\end{array}\right)=\left(\begin{array}{cc}
-D_{i} & \gamma_{i} \\
\lambda_{i} & e n_{e l} D_{e}
\end{array}\right) \cdot\left(\begin{array}{c}
\nabla n_{i} \\
\mathbf{E}
\end{array}\right),
$$

where $D_{i}$ is the polariton or exciton diffusion coefficient and $D_{e}$ is the mobility coefficient of the electrons. Only normal component in the polariton subsystem is dragged by the electron current, while the superfluid component is not dragged. Thus, the appearance of the polariton superfluidity can be detected by the electron-polariton drag effect.

Below in our calculations we assume that the exciton system is dilute, satisfying to the following condition $n_{e x} a_{0}^{2} \ll 1$, where $n_{e x}$ is the quasistationary concentration of excitons, created by the pumping, $a_{0}$ is the $2 \mathrm{D}$ exciton Bohr radius. The condition $n_{e x} a_{0}^{2} \ll 1$ holds for the excitons at the exciton densities up to $n_{e x} \approx 10^{12} \mathrm{~cm}^{-2}$, since in $\mathrm{GaAs} / \mathrm{GaAsAl}$ quantum well the exciton Bohr radius is in the order of $a_{0} \sim 10-50 \AA$. It is obvious to conclude that the system of polaritons is also dilute, since the system of excitons, that forms the polaritons, is dilute.

We obtain the drag coefficients $\gamma_{i}$ from the expansion of the polariton or exciton current $\mathbf{i}_{i}$ by keeping the leading term linear by the electric field $\mathbf{E}$. This current is given by

$$
\mathbf{i}_{i}=\frac{1}{M_{i}} \int \mathbf{p} n_{i}(\mathbf{p}) \frac{s d^{2} p}{(2 \pi \hbar)^{2}},
$$

where $\mathbf{p}$ is the polariton or exciton momentum, $M_{i}$ is the effective mass of polariton or mass of exciton, $s=4$ is the spin degeneracy, and $n(\mathbf{p})$ is the distribution function of the excitons or quasiparticle excitations in the polariton subsystem

$$
n_{i}(\mathbf{p})=n_{0 i}(\mathbf{p})\left[1+\left(1+n_{0 i}(\mathbf{p})\right) g(\mathbf{p})\right] .
$$

In (3) $g(\mathbf{p})$ is the contribution to the quasiparticle distribution function corresponding to the nonequilibrium correction due to the gradient of the chemical potential of polaritons (or excitons at high $T$ ) which was found by solving the kinetic equations. In (3) $n_{0 i}(\mathbf{p})$ is the BoseEinstein distribution function of the quasiparticles in the polariton or exciton subsystems at the equilibrium:

$$
\left.n_{0 i}(\mathbf{p})=\left(\exp \left(\left[\varepsilon_{i}(\mathbf{p})-\mu_{i}\right] /\left(k_{B} T\right)\right]\right)-1\right)^{-1} .
$$

In (44) the energy spectrum of the quasiparticles and chemical potential are given within the Bogoliubov approximation. However, for non-interacting excitons in (4) the single particle spectrum is $\varepsilon_{e x}=p^{2} /\left(2 M_{e x}\right)$. The effective mass of a polariton is

$$
M_{p}=2\left(M_{e x}^{-1}+\frac{c L_{C}}{\sqrt{\epsilon} \hbar \pi}\right)^{-1}
$$

where $M_{e x}=m_{e}+m_{h}$ is the exciton mass, $L_{C}$ is the length of optical microcavity, and $\epsilon$ is the dielectric constant (see, e.g, Ref. [18] and references therein).

In a many-particle system of excitons and electrons we replaced the bare pair exciton-electron interaction by the effective interaction $W_{\text {eff }}$ [19] corresponding to the exciton-electron interaction screened by the electronelectron interactions in 2DEG in the random phase approximation.

We have obtained the polariton distribution function (31) by solving the kinetic equations and using the exciton-electron interaction vertex in the Born approximation. By substituting it in (2), we find the following estimation for the drag coefficient $\gamma_{p}$ at low temperatures $k_{B} T \ll \mu$ and $k_{B} T \ll \hbar \Omega_{R}$ (we skip over the complicated derivations and present only the simple estimation for $\left.\gamma_{p}\right)$ :

$$
\gamma_{p}=\frac{1}{144}\left(\frac{21}{32}\right)^{2} \frac{\pi^{2} s^{2} e^{5} a_{0}^{6} \bar{\tau}_{n} \bar{\tau}_{e}}{\hbar^{3} a_{e}^{4} \epsilon^{2} D^{4}} \exp \left[-\mu_{p} /\left(k_{B} T\right)\right] .
$$

Note that the polariton relaxation time can be expressed in terms of the relaxation time of the normal (nonsuperfluid) component of exciton liquid. So in (6) we approximated the relaxation time of the normal component of exciton liquid and electron relaxation time by their average values $\tau_{n}$ and $\tau_{e}$, respectively. These average values can be obtained from the exciton mobility $\tilde{\mu}_{e x}=e \bar{\tau}_{n} / M$ and the electron mobility $\tilde{\mu}_{e}=e \bar{\tau}_{2} / m_{e}$ presented in Fig. 1 and Fig. 2 in Ref. [20]. Since derivation $\gamma_{p}$ resulting in (6) implies the condition $k_{B} T \ll \mu$ and $k_{B} T \ll \hbar \Omega_{R}$, we can apply (6) for the temperatures below $\sim 20 \mathrm{~K}$.

Let us mention that Eq. (6) was obtained by using the regular Bogoliubov approximation for the weaklyinteracting Bose gas with no dissipation [21]. We assume that the leakage of the photons from the microcavity is very small, and the system can be considered in the quasiequilibrium.

For high temperature, $k_{B} T \gtrsim \hbar \Omega_{R}$, the majority of polaritons occupy the upper polariton branch, where the upper polariton mass is very close to the mass of exciton $M_{e x}$. So at high temperature the polaritons are replaced by the free Bose gas of excitons [5].

We apply for the estimation of the exciton-electron drag coefficient for the dilute exciton system the same approach as for the polariton-electron drag coefficient for the dilute polariton system and present only the simple estimation:

$$
\begin{aligned}
\gamma_{e x} & =\frac{1}{144}\left(\frac{21}{32}\right)^{2} \frac{\pi^{2} s^{2} e^{5} a_{0}^{6} \bar{\tau}_{n} \bar{\tau}_{e}}{\hbar^{3} a_{e}^{4} \epsilon^{2} D^{4}} \\
& \times \exp \left[\left(k_{B} T-\mu_{e x}\right) /\left(k_{B} T\right)\right] .
\end{aligned}
$$

It is easy to see that at low temperatures in the very dilute systems of the polaritons the collective excitations described by the linear region in the spectrum give no contribution to $\lambda_{p}$. In the quasiparticle approach the 
normal component of the polariton system can be calculated by the integration of the Bose-Einstein distribution function (4) for the quasiparticles. Since $\lambda_{p}$ is directly proportional to $\partial \mu_{i} / \partial n_{i}$ [17], we obtain for the polariton subsystem

$$
\left(\frac{\partial n_{p}}{\partial \mu_{p}}\right)_{\mu_{p}=0}=\frac{s k_{B} T}{2 \pi \hbar^{2} c_{s}^{2}} \int_{0}^{\infty} \frac{e^{x} x d x}{\left(e^{x}-1\right)^{2}},
$$

where $c_{s}$ is the sound velocity for the quasiparticles in the polariton subsystem [18], and

$$
\left.\left(\frac{\partial n_{e x}}{\partial \mu_{e x}}\right)\right|_{\mu_{e x}=\mu_{e x}^{(0)}}=\frac{s M_{e x}}{2 \pi \hbar^{2}}\left[1-\exp \left[-\frac{2 \pi \hbar^{2} n}{s M_{e x} k_{B} T}\right]\right]
$$

for the ideal 2D Bose gas of excitons. The integral in the r.h.s. of (8) diverges. Therefore, we have $\partial n_{p} / \partial \mu_{p} \rightarrow \infty$, which results in $\lambda_{p}=0$ according to the fact that $\lambda_{p}$ is directly proportional to $\partial \mu_{i} / \partial n_{i}$ [17]. This result comes from the assumption that for the very dilute Bose gas of polaritons we took into account only the sound region of the collective excitation spectrum at small momenta, and neglect almost not occupied regions with the quadratic spectrum at large momenta and crossover dependence of the spectrum at the intermediate momenta. Our approximation results in suppressed $\lambda_{p}$. Therefore, in the presence of the superfluidity of polaritons, the polaritons moving due to their density gradient almost do not drag electrons and there is suppressed electron current induced by the polaritons. Hence, the suppression of the dragged electric current in the electron QW can indicate the superfluidity of the polaritons.

Figs. 1 and 2 present the results of calculations of the drag coefficients $\gamma_{p}$ and $\gamma_{e x}$, correspondingly, as a functions of temperature $T$ for the different interwell separation $D$. In our calculations we used the same interwell distances that used in the drag experiments [14, 23], namely $17.5 \mathrm{~nm}, 20 \mathrm{~nm}, 22.5 \mathrm{~nm}$ and $30 \mathrm{~nm}$. The calculations were performed for the exciton density $n=$ $10^{10} \mathrm{~cm}^{-2}$ with the parameters for the $\mathrm{GaAs} / \mathrm{GaAsAl}$ quantum wells: $m_{e}=0.07 m_{0}, m_{h}=0.15 m_{0}, M=$ $0.24 m_{0}, \epsilon=13$. All drag coefficients increase with the temperature $T$ and decrease with the interwell separation $D$ as $D^{-4}$.

We propose the following experiments relevant to electron-polariton and electron-exciton drag effect. The screen with two diaphragms covers the quantum well embedded into a semiconductor microcavity. The laser pumping through one diaphragm generates excitons forming the polaritons by coupling to the photons. At low temperature the absence of the electron current will indicate the superfluidity phase of the polaritons, while at high temperature the existence of the electron current will indicate the drag of electrons by the moving excitons. Therefore, the drag coefficient allows to estimate the dragged electron current. When the electron current is induced by the external electric field applied to the electron QW, the photoluminescence spectrum can be measured in the other diaphragm. At low temperature,

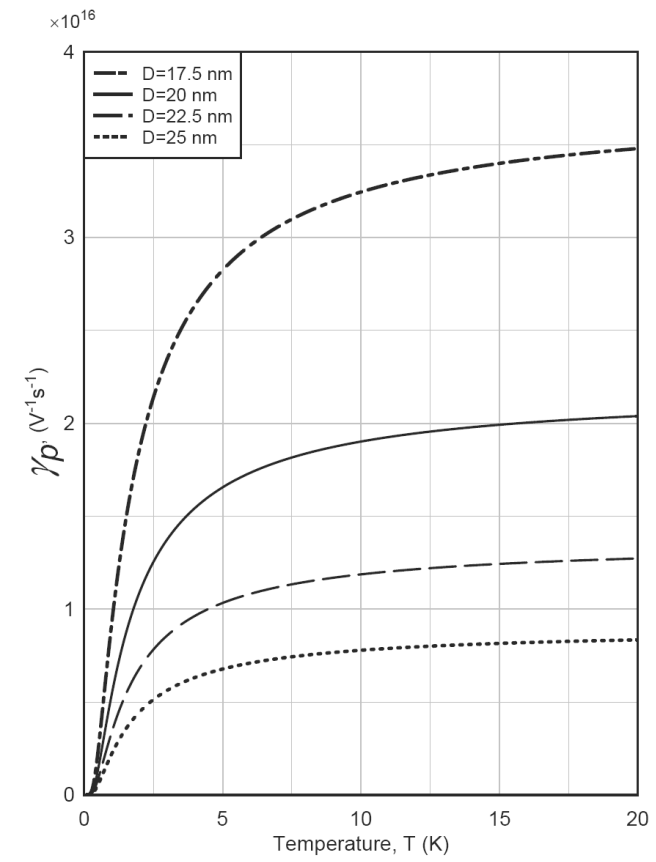

FIG. 1: The drag coefficient $\gamma_{p}$ for the system of spatially separated superfluid microcavity polaritons and electrons as a function of temperature $T$ for the different interwell separations $D$

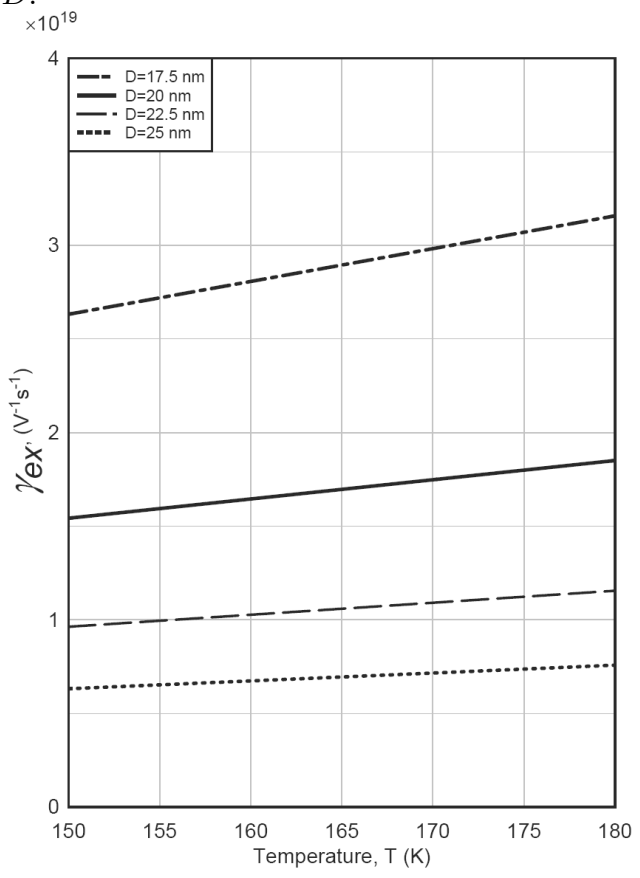

FIG. 2: The drag coefficient $\gamma_{e x}$ for the system of spatially separated excitons and electrons as a function of temperature $T$ for the different interwell separations $D$.

the difference between the photoluminescence spectrum of polaritons decay with the electric field applied to the electrons and without the electric field will indicates that the polaritons moved to the other place of the QW due to the drag by the electron current. The photolumines- 
cence spectrum in the other diaphragm without the electric field is caused only by the diffusion of the polaritons. Only the normal component of the polariton subsystem will move to the other diaphragm, while the superfluid component is not affected by the electrons. It seems like electrons move the photons that coupled with excitons. At high temperature the photoluminescence spectrum of the electron-hole recombination indicates that the excitons moved to the other place of the QW due to the drag by the electron current.

The other suggested experiment is based on the observation of the angle distribution of the photons escaping the optical microcavity. At low temperature we propose to create the uniform distribution of polaritons by the laser pumping within the microcavity. Therefore, $\nabla n_{p}=0$ and there is no polariton current. In the absence of polariton current the average angle of the photons escaping the optical microcavity and the perpendicular to the microcavity is $\bar{\alpha}=0$, because the angle distribution is symmetrical [4]. Let us induce the electron current by applying the electric field $\mathbf{E}$ and analyze the photon angle distribution in the presence of the nonzero current of polaritons along the quantum well parallel to the cavity entrained by the electron current due to the drag effect. If $\nabla n_{p}=0$ the polariton current is $\mathbf{i}_{p}=\gamma_{p} \mathbf{E}$, and according to the definition of polariton current we have $\mathbf{v}_{p}=\gamma_{p} \mathbf{E} / n_{p}$. Therefore, we can obtain the average component of the polariton momentum in the direction parallel to the Bragg mirrors of the microcavity: $\overline{p_{\|}}=M_{e f f} v_{p}=M_{e f f} \gamma_{p} E / n_{p}$. Since the perpendicular to the Brag mirrors component of the polariton momentum is given by $p_{\perp}=\hbar \pi / L_{C}$ [3] , we obtain for the average tangent of the angle between the path of the escaping photon and the perpendicular to the microcavity:

$$
\overline{\tan \alpha}=\frac{\overline{p_{\|}}}{p_{\perp}}=\frac{\gamma_{p} M_{p} L_{C} E}{\hbar \pi n_{p}} .
$$

Note that only normal (non-superfluid) component of the polariton subsystem will contribute to the drag coefficient $\gamma_{p}$ and to $\overline{\tan \alpha}$. There will be two peaks of the escaping photons: one at $\overline{\tan \alpha} \neq 0$ corresponds to the moving (dragged) normal component, and the other one at $\overline{\tan \alpha}=0$ corresponds to the superfluid component. The analysis of the angle distributions of the photons escaping the optical microcavity has been used in the experiments [8, 9].

Let us make estimations of the parameters for the drag effects. At the temperature $T=4 \mathrm{~K}$ the experiment [4, 7] shows that the polariton lifetime $\tau=10 \mathrm{ps}$, and the polariton diffusion path is $l=20 \mu \mathrm{m}$. The corresponding average polariton velocity is $v_{p}=l / \tau=2 \times 10^{6} \mathrm{~m} / \mathrm{s}$. Since $E=n_{p} v_{p} / \gamma_{p}$, we can estimate the electric field $E$ corresponding to such drag effect. For $n_{p}=10^{10} \mathrm{~cm}^{-2}$ and $T=4 \mathrm{~K}$ from Fig. 1 for the interwell separation $D=17.5 \mathrm{~nm} \gamma_{p}=2.64 \times 10^{16} \mathrm{~V}^{-1} \mathrm{~s}^{-1}$, the corresponding electric field is $E=3.8 \times 10^{3} \mathrm{~V} / \mathrm{m}$ which corresponds to the applied voltage $V=3.8 \times 10^{-3} \mathrm{~V}$ at the size of the system $d=1 \mu \mathrm{m}$. Using $M_{p}=7 \times 10^{-5} \times m_{e}$, the length of the microcavity $L_{C}=2 \mu \mathrm{m}$ [4, 7], and the estimated $\gamma_{p}$ and $E$ in (10), we obtain for the average tangent of the angle $\alpha: \overline{\tan \alpha}=0.385$ and $\tan ^{-1}(\overline{\tan \alpha})=21^{0}$.

We can conclude that at low temperatures the electron current dragged by the polariton current is strongly suppressed. However, the polariton current can be dragged by the electrons, and, therefore, there is a transport of photons along the microcavity, which can be observed through the angular distribution of photons discussed above. At high temperatures, from one side, the existence of the electric current in an electron QW indicates the exciton current in the other QW, from the other side, the electron current in one QW induces the exciton current in the other QW via the drag of excitons by the electrons. The obtained drag coefficients allow calculate the corresponding currents in the presence of the superfluid component. The suggested experiments allow to observe the electron-cavity polariton drag effects and to observe directly the cavity polariton superfluidity.

\section{Acknowledgments}

O. L. B., R. Ya. K. were supported by PSC CUNY grant 63443-0041, and Yu. E. L. was partially supported by RFBR and RAS programs.
[1] V. M. Agranovich, Excitations in Organic Solids, (Oxford University Press, Oxford, England, 2009).

[2] A. Kavokin and G. Malpeuch, Cavity Polaritons (Elsevier, 2003).

[3] D. W. Snoke, Solid State Physics. Essential Concepts. (Addison-Wesley, 2008).

[4] R. Balili, V. Hartwell, D. W. Snoke, L. Pfeiffer and K. West, Science 316, 1007 (2007).

[5] P. Littlewood, Science 316, 989 (2007).

[6] J. Kasprzak, et. al., Nature 443, 409 (2006).

[7] R. Balili, B. Nelsen, D. W. Snoke, L. Pfeiffer, and K. West, Phys. Rev. B 79, 075319 (2009).

[8] A. Amo, et al. Nature Phys. 5, 805 (2009).
[9] A. Amo, et al., Nature 457, 291 (2009).

[10] Here we omit weak effects of direct nonresonant interaction of photons with $2 \mathrm{D}$ electron gas which will be considered elsewhere.

[11] Yu. E. Lozovik and V. I. Yudson, JETP Lett. 22, 26 (1975).

[12] M. B. Pogrebinskii, Sov. Phys. Semicond. 11, 372 (1977).

[13] P. J. Price, Physica B 117 \& 118, 750 (1983).

[14] T. J. Gramila, J. P. Eisenstein, A. H. MacDonald, L. N. Pfeiffer, and K. W. West, Phys. Rev. Lett. 66, 1216 (1991), Phys. Rev. B 47, 12957 (1993).

[15] G. Vignale and A. H. MacDonald, Phys. Rev. Lett. 76 2786 (1996). 
[16] L. E. Gurevich, J. Phys. (USSR) 9, 477 (1945); J. Phys. (USSR) 10, 67 (1946).

[17] Yu. E. Lozovik and M. V. Nikitkov, JETP 89, 775 (1999).

[18] O. L. Berman, Yu. E. Lozovik, and D. W. Snoke, Phys. Rev. B 77, 155317 (2008).

[19] D. V. Kulakovskii and Yu. E. Lozovik, JETP 98, 1205 (2004).

[20] W. Walukiewicz, H. E. Ruda, J. Lagowski, and H. C. Gatos, Phys. Rev. B 30, 4571 (1984).

[21] If the exciton-polaritons have a finite life-time in the cavity, the Bogoliubov dispersion is modified at small wave vectors [22]. This modification should affect the drag. However, we consider the small relative deviation from the threshold pumping intensity, when the pumping maintains the exact balance of amplification and losses. According to Eq. (6) in Ref. [22], at small relative deviation from the threshold pumping intensity, the spectrum of collective excitations in the system of exciton polaritons corresponds to the regular Bogoliubov approximation.

[22] M. Wouters and I. Carusotto, Phys. Rev. Lett. 99, 140402 (2007).

[23] J. A. Seamons, C. P. Morath, J. L. Reno, and M. P. Lilly, Phys. Rev. Lett. 102, 026804 (2009). 